\title{
3 Research Square \\ Female Versus Male Oral Cavity Cancer: is There a Difference?
}

\section{Yi-Chieh Lee}

Chang Gung Memorial Hospital

\section{Huei-Tzu Chien}

Chang Gung University of Science and Technology

Chi-Kuang Young

Keelung Chang Gung Memorial Hospital of the CGMF

\section{Shy-Chyi Chin}

Chang Gung Memorial Hospital

\section{Andrea landelli}

Chang Gung Memorial Hospital

Chun-Ta Liao

Chang Gung Memorial Hospital

\section{Chung-Kang Tsao}

Chang Gung Memorial Hospital

\section{Chung-Jan Kang}

Chang Gung Memorial Hospital

\section{Shiang-Fu Huang ( $\square$ bigmac@adm.cgmh.org.tw )}

Chang Gung Memorial Hospital Linkou Branch https://orcid.org/0000-0003-3582-9938

\section{Research}

Keywords: female, male, oral cavity cancer, survival

Posted Date: August 5th, 2020

DOI: https://doi.org/10.21203/rs.3.rs-53503/v1

License: (c) (i) This work is licensed under a Creative Commons Attribution 4.0 International License.

Read Full License 


\section{Abstract}

Objectives:

Oral cavity squamous cell carcinoma (OSCC) is a leading cause of death in Taiwan, and most of the patients are male. Little is known about the differences in risk factors, cancer characteristics and treatment outcomes in female patients. The study aim is to investigate the clinicopathological and outcome differences between gender in patients affected by oral cancer in Taiwan.

\section{Methods:}

This is a retrospective study based on data obtained between 1995 and 2019. A total of 2,046 patients were recruited for analysis. Cancer characteristics, risk factors and treatment outcomes in patients with oral cancer between genders were collected.

\section{Results:}

Female patients represented the $6.7 \%$ of the entire cohort of study. Females were diagnosed at an older age and at an earlier local stage compared to male patients $(p<0.001)$. The female patients were less exposed to cigarette, alcohol, and betel-nut (BQ) (all p-values $<0.001)$. Tongue $(55.1 \%)$ was the most frequent subsite involved in the female group, while buccal (38.4\%) and tongue (35.3\%) were more likely $(p<0.001)$ to be associated with male gender. In tongue cancer subgroup, female patients presented less frequently extra-nodal extension compared with male patients $(p=0.040)$. During the follow-up period, there was no significant difference in recurrence and overall deaths between genders.

\section{Conclusion:}

In Taiwan, the male to female ratio in OSCC is 14:1. The tumor subsite distribution, environment exposure and stage distribution are different between females and male. There are no differences in term of survival between female and male OSCC patients.

\section{Introduction}

Oral cancer is the sixth most prevalent cancer worldwide and determines a leading cause of death in Taiwan, especially in the male population.[1] As estimated by the Ministry of Health and Welfare of Taiwan, oral cancer has remained the top five causes of death for years. [2] The incidence is about 7,000 cases annually in Taiwan, which led to 3,027 deaths in 2018. Among the deaths, 2,779 were male, accounting for $90 \%$ of the mortality caused by oral cancer.[2] Oral cancer was observed to affect the male more than the female globally, and the ratio was highest in Taiwan (male to female ratio: 10.5).[3] In the male patients in Taiwan, the consumption of cigarettes, alcohol and betel quid (BQ) predisposes them to develop oral cavity cancer. It is estimated that cigarettes and alcohol attributed to about $80 \%$ of oral cancer. [1] In Taiwan, the significant sex- related differences in the frequency of tobacco and betel quid 
chewing may explain the higher incidence of oral cavity SCC in males (20.81 cases per 1 million persons) than in females (2.40 cases per 1 million persons) [4]

Due to the substantially lower number of female patients with oral cancer, most of the studies focused on the male population. Little is known about the differences in risk factors attributing to oral cancer and treatment outcomes in women. For female patients, the environmental carcinogens' exposure is much less frequent in BQ endemic regions such as Taiwan and India. Some studies indicated that oral cancer might have different risk factors between genders.[5, 6] Nevertheless, one study conducted in Italy and Switzerland showed that tobacco smoking and alcohol consumption represents the significant risk factors for both women and men; furthermore, Honorato et al. reported no significant differences in survival rates between genders. [7, 8] Therefore, Considering the lack of general agreement in the literature, this study aimed to investigate the differences in characteristics, risk factors, and treatment outcomes between females and males patients affected by OSCC in Taiwan.

\section{Methods And Materials:}

\section{Study design}

This retrospective study was based on data obtained from a single medical center (Chang Gung Memorial Hospital, Linkou branch, Taiwan). The data consisted of 2,046 participants diagnosed with oral cavity cancer (involving tongue, buccal, hard palate, gingiva, and floor of mouth) between Jul 1995 and Mar 2019. All the patients received pre-operative MRI, chest X-ray, liver sonography, and bone scan/ positron emission tomography (PET). All the patients had radical surgery as their first treatment. Clinical data were collected from medical records. The staging system was based on American Joint Committee on Cancer TNM staging system, (AJCC, 2010 edition).[9] After radical surgeries, tumors with adverse pathologic factors such as advanced tumor stage (T3 or T4), poor tumor differentiation, lymph node extranodal spread (ENE), or tumor depth $\geq 10 \mathrm{~mm}$ underwent adjuant radiotherapy or chemoradiotherapy.[10,11] Patients were included if they had complete information and patients with distant metastasis or recurrence carcinoma were excluded in the study.

\section{Data collection}

Tobacco, alcohol, and BQ consumption history was divided into two groups: never use and ever/current use. Cancer sites included tongue, mouth floor, lip, buccal, alveolus (gum), hard palate, and retromolar trigone. The pathologic parameters included tumour size, tumour depth, perineural invasion, lymph node metastasis. Lymph node status was further classified as three categories, including negative, positive lymph node metastasis without ENE, and positive lymph node metastasis with ENE. Tumor size was classified as "small" if the patient had tumor in T1/T2 and "large" as tumor in T3/T4. Overall cancer stage in I and II was classified as early cancer and stage III/IV was classified as "advanced". All the patients were regularly followed up after the surgery. They returned to clinic every month in the first year, 
2-month in the second year, 3-month in the third year, 4-month in the fourth year, and 6 monthly after 5 years.

\section{Statistical analysis}

Statistical analysis was conducted to explore the dataset and compare the baseline characteristics between males and females. To assess the differences, chi-squared $\left(\chi^{2}\right)$ test was used for categorical characteristics and t-test was used for continuous variables. All $p$ values $<0.05$ were regarded as statistical significance. Overall and disease-free survival were assessed by using the Kaplan-Meier method and the differences were estimated by log-rank test. All analyses were conducted in STATA (StataCorp LLC, Texas, USA) version 15.

\section{Results}

\section{Patient characteristics}

Table 1 showed the distribution of baseline characteristics. A total of 2,046 observations met the inclusion criteria and were included for further analysis. The study population consisted of 1,910 males (93.4\%) and 136 females (6.6\%). The male-to-female ratio was 14:1 in the study. The average age of participants was 50.7 years old (Standard deviation: 11.2, range: 24.0 to 92.0.) Most of the participants had a history of smoking (84.7\%), alcohol (72.1\%), and BQ chewing (79.9\%). Cancer sites were more frequent in tongue (35.3\%) and buccal mucosa (38.4\%). More than half of participants $(58.1 \%)$ were diagnosed at an advanced stage. During the follow-up period, 649 (31.7\%) participants were found to have recurrence. 
Table 1

Baseline patient characteristics

\begin{tabular}{|c|c|c|c|}
\hline & & $\mathbf{N}(\%)$ & Standard error \\
\hline \multirow[t]{2}{*}{ Gender } & Male & $1,910(93.4)$ & \\
\hline & Female & $136(6.7)$ & \\
\hline Age & & 50.7 & 11.2 \\
\hline \multirow[t]{2}{*}{ Smoking } & Never & $313(15.3)$ & \\
\hline & Yes & $1,733(84.7)$ & \\
\hline \multirow[t]{2}{*}{ Alcohol } & Never & $570(27.9)$ & \\
\hline & Yes & $1,476(72.1)$ & \\
\hline \multirow[t]{2}{*}{ Betel-nut } & Never & $411(20.1)$ & \\
\hline & Yes & $1,635(79.9)$ & \\
\hline \multirow[t]{7}{*}{ Cancer site } & Tongue & $723(35.3)$ & \\
\hline & Mouth Floor & $72(3.5)$ & \\
\hline & Lip & $70(3.4)$ & \\
\hline & Buccal & $786(38.4)$ & \\
\hline & Gum & $237(11.6)$ & \\
\hline & Hard Palate & $49(2.4)$ & \\
\hline & Retromolar & 109(5.3) & \\
\hline \multirow[t]{3}{*}{ Stage_N stage (ECS) } & N- ECS- & $1,273(62.2)$ & \\
\hline & $\mathrm{N}+\mathrm{ECS}-$ & $313(15.3)$ & \\
\hline & $\mathrm{N}+\mathrm{ECS}+$ & $460(22.5)$ & \\
\hline \multirow[t]{2}{*}{ Stage_T } & Early & $1,228(60.0)$ & \\
\hline & Late & $818(40.0)$ & \\
\hline \multirow[t]{2}{*}{ Stage_overall } & Early & $857(41.9)$ & \\
\hline & Advanced & $1,189(58.1)$ & \\
\hline \multirow[t]{2}{*}{ Recurrence } & Yes & $649(31.7)$ & \\
\hline & No & $1,397(68.3)$ & \\
\hline
\end{tabular}


Table 2 showed the comparison of characteristics between genders. Male patients were diagnosed with oral cancer at their younger age (50.2; S.D.: 10.90) compared to female patients (57.1; S.D.: 13.11, $\mathrm{p}<$ 0.001). The consumption of cigarettes, alcohol, and BQ varied between genders. The majority of male patients had a history of consumption of cigarettes, alcohol, and betel-nut whereas the female patients did not (Table 2 , all $p$ values $<0.001)$. 
Table 2

Baseline patient characteristics between genders and $p$-value $(N=2046)$

\begin{tabular}{|c|c|c|c|c|}
\hline & & $\begin{array}{l}\text { Male } \\
\mathrm{N}(\%)\end{array}$ & $\begin{array}{l}\text { Female } \\
\mathrm{N}(\%)\end{array}$ & p-value \\
\hline & & $1910(93.4)$ & $136(6.7)$ & \\
\hline Age & & $50.2(49.7-50.7)$ & $57.1(54.9-59.4)$ & $<0.001$ \\
\hline Smoking & Never & $214(11.2)$ & $99(72.8)$ & $<0.001$ \\
\hline & Yes & $1696(88.8)$ & $37(27.2)$ & \\
\hline Alcohol & Never & $464(24.3)$ & $106(77.9)$ & $<0.001$ \\
\hline & Yes & $1446(75.7)$ & $30(22.1)$ & \\
\hline Betel-nut & Never & $313(16.4)$ & $98(72.1)$ & $<0.001$ \\
\hline & Yes & $1597(83.6)$ & $38(27.9)$ & \\
\hline Cancer site & Tongue & $648(33.9)$ & $75(55.1)$ & $<0.001$ \\
\hline & Mouth floor & $68(3.6)$ & $4(2.9)$ & \\
\hline & Lip & $64(3.1)$ & $6(4.4)$ & \\
\hline & Buccal & $760(39.8)$ & $26(19.1)$ & \\
\hline & Gum & $219(11.5)$ & $18(13.2)$ & \\
\hline & Hard palate & $46(2.4)$ & $3(2.2)$ & \\
\hline & Retromolar & $105(5.5)$ & $4(0.2)$ & \\
\hline Stage_N (ENE) & N-ENE- & $1190(62.3)$ & $83(61.0)$ & 0.557 \\
\hline & $\mathrm{N}+\mathrm{ENE}-$ & $288(15.1)$ & $25(18.4)$ & \\
\hline & $\mathrm{N}+\mathrm{ENE}+$ & $432(22.6)$ & $28(20.6)$ & \\
\hline Stage_T & Early & $1127(59.0)$ & $101(74.3)$ & $<0.001$ \\
\hline & Late & $783(41.0)$ & $35(25.7)$ & \\
\hline Stage_overall & Early & $793(41.5)$ & $64(47.1)$ & 0.206 \\
\hline & Advanced & $1117(58.5)$ & $72(52.9)$ & \\
\hline Recurrence & Yes & $613(32.1)$ & $36(26.5)$ & 0.173 \\
\hline & No & $1,297(67.9)$ & $100(73.5)$ & \\
\hline
\end{tabular}


The occurrence of cancer sites was also observed to vary between genders with a significant difference $(p<0.001)$. The most common cancer site in the male patients was the buccal $(39.8 \%)$ followed by tongue (33.9\%). Unlike the male patients, more than half of female patients were diagnosed to have tongue cancer (55.1\%) which was followed by buccal cancer (19.1\%). The diagnosed disease overall stage was similar between genders: $41.5 \%$ was early stage in males and $47.1 \%$ in females $(p=0.206)$. During the follow-up period, the male patients were observed to have about one-third (32.1\%) recurrence rate compared to one-quarter $(26.5 \%)$ recurrence rate in female patients. It did not reach statistical significant difference evidence $(p=0.17)$ between genders.

We further analyzed in patients with tongue cancer since tongue was the most common affected anatomical site both in male (33.9\%) and female patients (55.1\%). The disease characteristics and treatment outcomes were compared between genders. Table 3 showed the comparison between genders in patients with tongue cancer. The females accounted for about $10 \%$ of the tongue cancer, which was similar to the gender distribution in all oral cancer patients. Females were about 5 years $(53.6,95 \% \mathrm{Cl}$ : $50.6-56.6)$ older when diagnosed with tongue cancer than males (48.4, 95\% Cl: $28.0-49.6)$. As is all oral cancer patients, most males were heavy use of tobacco, alcohol, and BQ whereas the females were not. More females (93.3\%) with tongue cancer were diagnosed at an early tumor status and were significantly different compared with $73.5 \%$ of males diagnosed at an early status $(p<0.001)$. For the lymph node metastasis, the proportion of those without lymph node involvement was similar between genders in tongue cancer. However, the females had fewer ENE if they had lymph node involvement compared with those of males ( $\mathrm{N}+\mathrm{ENE}+$, female: $13.3 \%$; male: $24.9 \%, \mathrm{p}=040$ ). For the overall stage, the difference between genders was not significant $(p=0.07)$. 
Table 3

Baseline patient characteristics between genders and $\mathrm{p}$-value in tongue cancer $(\mathrm{N}$ $=723$ )

\begin{tabular}{|c|c|c|c|c|}
\hline & & Male & Female & p-value \\
\hline & & $\mathrm{N}(\%)$ & $\mathbf{N}(\%)$ & \\
\hline & & 648 (89.6) & 75 (10.4) & \\
\hline Age & & $48.4(28.0-49.6)$ & $53.6(50.6-56.6)$ & $<0.001$ \\
\hline Smoking & Never & $86(13.3)$ & $57(76.0)$ & $<0.001$ \\
\hline & Yes & $562(86.7)$ & $18(24.0)$ & \\
\hline Alcohol & Never & $158(24.4)$ & $59(78.7)$ & $<0.001$ \\
\hline & Yes & $490(75.6)$ & $16(21.3)$ & \\
\hline Betelnut & Never & 118 (18.2) & $60(80.0)$ & $<0.001$ \\
\hline & Yes & $530(81.8)$ & $15(20.0)$ & \\
\hline Stage_N (ENE) & N-ENE- & $390(60.2)$ & $48(64.0)$ & 0.040 \\
\hline & $\mathrm{N}+\mathrm{ENE}-$ & $97(15.0)$ & $17(22.7)$ & \\
\hline & $\mathrm{N}+\mathrm{ENE}+$ & 161 (24.9) & $10(13.3)$ & \\
\hline Stage_T & Early & $476(73.5)$ & 70 (93.3) & $<0.001$ \\
\hline & Late & $172(26.5)$ & $5(6.7)$ & \\
\hline Stage_overall & Early & $325(50.2)$ & $46(61.3)$ & 0.067 \\
\hline & Advanced & 323 (49.9) & $29(38.7)$ & \\
\hline Recurrence & Yes & $454(70.1)$ & 55 (73.3) & 0.557 \\
\hline & No & 194 (29.9) & $20(26.7)$ & \\
\hline
\end{tabular}

\section{Disease free survival (DFS) and overall survival (OS)}

A total of 1979 with complete information on the time of diagnosis and death and 509 deaths among them were related with oral cancer. Four hundred and eighty males $(7.7 \%)$ and $29(7.5 \%)$ females expired during follow-up. On the other hand, 624 patients with recurrences had been observed. Half of the recurrence occurred within 8.79 years after being treated. Of the patients with recurrence, 589 were male and 35 were female. The frequency of recurrence among the female population was $9.4 \%$ and $10.5 \%$ in the male. Figure 1 showed Kaplan-Meier OS curves and Fig. 2 showed the DFS curves between genders There is weak evidence of a difference in OS between females and males during the follow-up period (log- 
rank test: $p=0.491)$. There was also no difference in the DFS between genders (log rank test: 0.280$)$. In tongue cancer, the most frequent site of OSCC in female, the DFS was not different between female and male $(p=0.336)$. However, OS in female tongue cancer was better than male but not reached statistical significance ( $p=5$-year OS: $91.6 \%$ vs $87.1 \%, p=0.074)$. Multivariate analysis adjusting age, tumor status and lymph node metastasis in DFS and OS showed no gender differences (DFS: $p=0.715$ and OS: $p=$ $0.170)$.

\section{Discussion}

The study included 2,046 patients in a single medical center and was wide in case number for analysis. However, it overlapped different staging systems (6th, 7th and 8th AJCC editions). We adopted the 7th edition throughout this study. In the study, more than $90 \%$ of the participants were male. They were more likely to be diagnosed with oral cancer at a younger age and with late tumor status compared with female patients. This age difference can be explained two folds: first, in betel endemic area, patients develop OSCC at a younger age, compared to other populations not exposed at this risk factor [1] [12] Second, the female patients were rarely exposed to cigarette, alcohol, and BQ, unlike the male patients, therefore, other factors, as poor oral hygiene, inadequate dental status and chronic irritation, which represents independent risk factors for OSCC, irrespective of tobacco and alcohol consumption, may have played a role in the carcinogenesis [13]. Not smoking, not drinking patients has tend to presents at the extremes of age [14]. It is reasonable that with less carcinogen exposure, female population develop oral cancer later in their lives.

Tongue $(55.1 \%)$ was the most frequent present anatomical site of oral cancer in female patients; this finding well correlates with the current literature; several studies had reported a higher involvement rate of this subsite, especially in non-smoker non-drinker women with HPV-negative OSCC [15]. On the other hand, we notice a slightly higher median age of our cohort, compared to other study [16]. We interpreted this discrepancy as to the results of the differences in the epidemiological basin assessed. Most of the studies are conducted in western countries, and low OSCC prevalence areas, as a matter of fact, Lin et al [17] which investigated the clinicopathological features in the Taiwanese population affected by OSCC have obtained similar results regarding the female patients' age. Furthermore, recent findings, carried by Foy et al. in France have advocated a possible relationship between the development of OSCC in nonsmoking, non-drinking patients and viral infection, hypothesizing that changes in western country sexual behaviors may lead to an increased incidence of herpes virus in the oral cavity, especially HSV-2, similarly to what has already been described in HPV-positive oropharyngeal squamous cell carcinoma. Because viral genome integration has not been detected in non-smoking and non-drinking OSCC, a "hit and run" viral mechanism involving epigenome deregulation could therefore play a key role at early steps of oral carcinogenesis in this population of patients.[18] In our cohort, males OSCC were more likely to occur in buccal (38.4\%) and tongue (35.3\%) locations. The buccal mucosa is the most affected site in people with BQ chewing history due to frequent mucosa irritation. Since most of the male patients $(79.9 \%)$ had BQ chewing history, buccal cancer was more likely to be found in the male participants. The tumor stage while diagnosed differs between genders. Most (74.3\%) of the females had early tumor stage while 
diagnosed with oral cancer whereas the male patients only had about $60 \%$ early tumor stage. However, the lymph node status and the overall stage did not vary between genders. When we did a further analysis in patents with tongue cancer, we found that female patients were diagnosed at an older age and with early tumor size. Besides, in lymph node involvement, the females had fewer ENE if they had lymph node involvement compared to the males ( + ENE+, male: $24.9 \%$; female: $13.3 \%)$. In non-smoking and non-drinking OSCC, the frequency of lymph node metastasis and ENE was less frequent than the smoking and drinking group. Although non-significant, the patients that smoked cigarettes and drank alcohol tend to have higher risks of lymph node ENE.[19] It could be related with the mechanisms of tumorigenesis. Those oral cancers related with smoking and BQ could harbour more genetic alterations from the environment carcinogens exposure and can be easier to metastasize or have ENE. The other possibility is the tongue cancers in male are more advanced in stage. The accumulated mutations with tumor stage make tumor cells aggressive in behaviour easier to metastasize.

During the follow-up period, there was no evidence of a difference in recurrence and deaths between genders. Since oral cancer was prevalent in the male population, most of the literature investigated the characteristics, risk factors, treatment, and outcomes of oral cancer in the male population. Few studies investigated this disease in females. We reviewed the studies about oral cancer between genders. Honorato et al.[8] compared the prognostic differences in oral cancer between genders in Brazil. The data showed similar consumption habits of alcohol and cigarette between genders with our population, whereas the anatomical sites of cancer differ from our experiences. It would be due to the lack of using BQ. The tongue was the most affected site in both males and females.[8] Due to the BQ consumption culture in Taiwan, buccal is the most affected site in oral cancer patients in Taiwan, unlike the results showed in Brazil. A study in European countries presented the risk factors of oral and pharyngeal cancer in women in Italy and Switzerland.[7] The study showed that women shared the same major risk factors such as habits of using cigarette and alcohol as men.

The rate of HPV infection in oral cavity cancer was about $13.4 \%$ while the infection rate was $36.7 \%$ in oropharyngeal cancer in the United States.[20] The HPV infection rate in OSCC is lower than the oropharyngeal cancer. In female OSCC patients, the frequency of HPV infection (9.6\%) was much lower than the male (15.8\%). In the large cohort study, female OSCC patients had better OS regardless of HPV status. It is the reason we speculated that the survival rates in our study would not be significantly influenced as the HPV status was not determined in in our analysis.

In our study, there were 2,046 participants included, while only 136 patients were female. However, this is one of the largest study recruited the most number of female OSCC underwent surgery as primary treatment. HPV-related head and neck cancer were noted to be increasing in recent years especially in the younger population and those without exposure to tobacco and alcohol. Recently, with the increasing use of smoking in female population. This study provides an epidemiologic data in female OSCC in an BQ endemic area. Further cohort studies at different time periods is mandatory to compare the trends for female OSCCs. 


\section{Conclusions}

In this study, we demonstrated that, in Taiwan, the male to female ratio in OSCC was 14:1. The cigarette smoking, alcohol and BQ were significantly less used in female patients. The tumor sub-site distribution was most commonly occurred in tongue. The tumor stage was early in diagnosis in the female patients. Female OSCC did not have worse prognosis than male from our analysis.

\section{Declarations}

\section{Ethics approval and consent to participate}

All research was carried out in compliance with the Helsinki Declaration. This study was approved by the institutional review board of the Chang Gung Memorial Hospital. [IRB No.: 202000599B0]

\section{Consent for publication}

The data in this article is not reporting the individual participant's identity and no permission are requested from the patients.

\section{Availability of data and materials}

The datasets used and analysed during the current study are available from the corresponding author on reasonable request.

\section{Competing interests}

The authors declare no conflict of interest.

\section{Funding}

This study was supported by grants CMRPG3H0793, CMRPG3J0591, CMRPG3J0592 and CMRPB53 from Chang Gung Memorial Hospital and grant MOST106-2314-B-182-025-MY3 and MOST 109-2314-B182-015- from the Ministry of Science and Technology, Executive Yuan, Taiwan, ROC and by the Health and Welfare Surcharge on Tobacco Products (grant MOHW109-TDU-B-212-134016) from the Ministry of Health and Welfare (MOHW), Executive Yuan, Taiwan, ROC.

\section{Authors' contributions}

YCL and SFH conceived the idea for the manuscript, conducted a literature search, and drafted the manuscript. YCL, HTC and SFH organized the manuscript and critically revised the manuscript. CKY, SCC, 
Al, CTL, CKT and CJK collected the data. YCL, HTC and CKY analysed the data. All the authors read and approved the final manuscript.

\section{Acknowledgements}

The authors thank all of the members of the Cancer Center, Chang Gung Memorial Hospital, Linkou, Taiwan, for their invaluable help.

\section{References}

1. Warnakulasuriya S. Global epidemiology of oral and oropharyngeal cancer. Oral Oncol. 2009;45:309-16.

2. Cause of Death Statistics. In. 2017.

3. Krishna Rao SV, Mejia G, Roberts-Thomson K, Logan R. Epidemiology of oral cancer in Asia in the past decade-an update (2000-2012). Asian Pac J Cancer Prev. 2013;14:5567-77.

4. Liao CT, Wen YW, Yang LY, et al. Comparative clinical outcomes of Taiwanese patients with resected buccal and tongue squamous cell carcinomas. Oral Oncol. 2017;67:95-102.

5. Chen F, He BC, Yan LJ, et al. Influence of oral hygiene and its interaction with standard of education on the risk of oral cancer in women who neither smoked nor drank alcohol: a hospital-based, casecontrol study. Br J Oral Maxillofac Surg. 2017;55:260-5.

6. Llewellyn CD, Linklater $\mathrm{K}$, Bell J, et al. An analysis of risk factors for oral cancer in young people: a case-control study. Oral Oncol. 2004;40:304-13.

7. Bosetti C. NE, Franceschi S, Conti E, Levi F, Tomei F, La Vecchi C. La. Risk factors for oral and pharyngeal cancer in women. a study from Italy and Switzerland. British Journal of Cancer 2000; 82.

8. Honorato J, Rebelo MS, Dias FL, et al. Gender differences in prognostic factors for oral cancer. Int J Oral Maxillofac Surg. 2015;44:1205-11.

9. FL G, DL P. ID F et al. AJCC cancer staging manual. 6th ed. New York: Springer-Verlag; 2002.

10. Huang S-F, Kang C-J, Lin C-Y, et al. Neck treatment of patients with early stage oral tongue cancer. Cancer. 2008;112:1066-75.

11. Liao C-T, Chang JT-C, Wang H-M, et al. Analysis of Risk Factors of Predictive Local Tumor Control in Oral Cavity Cancer. Ann Surg Oncol. 2007;15:915-22.

12. Su CC, Yang HF, Huang SJ, Lian le B. Distinctive features of oral cancer in Changhua County: high incidence, buccal mucosa preponderance, and a close relation to betel quid chewing habit. J Formos Med Assoc. 2007;106:225-33.

13. Rosenquist K, Wennerberg J, Schildt EB, et al. Oral status, oral infections and some lifestyle factors as risk factors for oral and oropharyngeal squamous cell carcinoma. A population-based casecontrol study in southern Sweden. Acta Otolaryngol. 2005;125:1327-36. 
14. Dahlstrom KR, Little JA, Zafereo ME, et al. Squamous cell carcinoma of the head and neck in never smoker-never drinkers: a descriptive epidemiologic study. Head Neck. 2008;30:75-84.

15. Brown LM, Check DP, Devesa SS. Oral cavity and pharynx cancer incidence trends by subsite in the United States: changing gender patterns. J Oncol 2012; 2012: 649498.

16. Satgunaseelan L, Allanson BM, Asher R, et al. The incidence of squamous cell carcinoma of the oral tongue is rising in young non-smoking women: An international multi-institutional analysis. Oral Oncol. 2020;110:104875.

17. Lin NC, Hsu JT, Tsai KY. Difference between Female and Male Patients with Oral Squamous Cell Carcinoma: A Single-Center Retrospective Study in Taiwan. Int J Environ Res Public Health 2020; 17.

18. Foy JP, Bertolus C, Boutolleau D, et al. Arguments to Support a Viral Origin of Oral Squamous Cell Carcinoma in Non-Smoker and Non-Drinker Patients. Front Oncol. 2020;10:822.

19. DeAngelis A, Breik $O, K o o ~ K$, et al. Non-smoking, non-drinking elderly females, a 5 year follow-up of a clinically distinct cohort of oral squamous cell carcinoma patients. Oral Oncol. 2018;86:113-20.

20. Li H, Park HS, Osborn HA, Judson BL. Sex differences in patients with high risk HPV-associated and HPV negative oropharyngeal and oral cavity squamous cell carcinomas. Cancers of the Head Neck. 2018;3:4.

\section{Figures}

\section{Figure 1.}

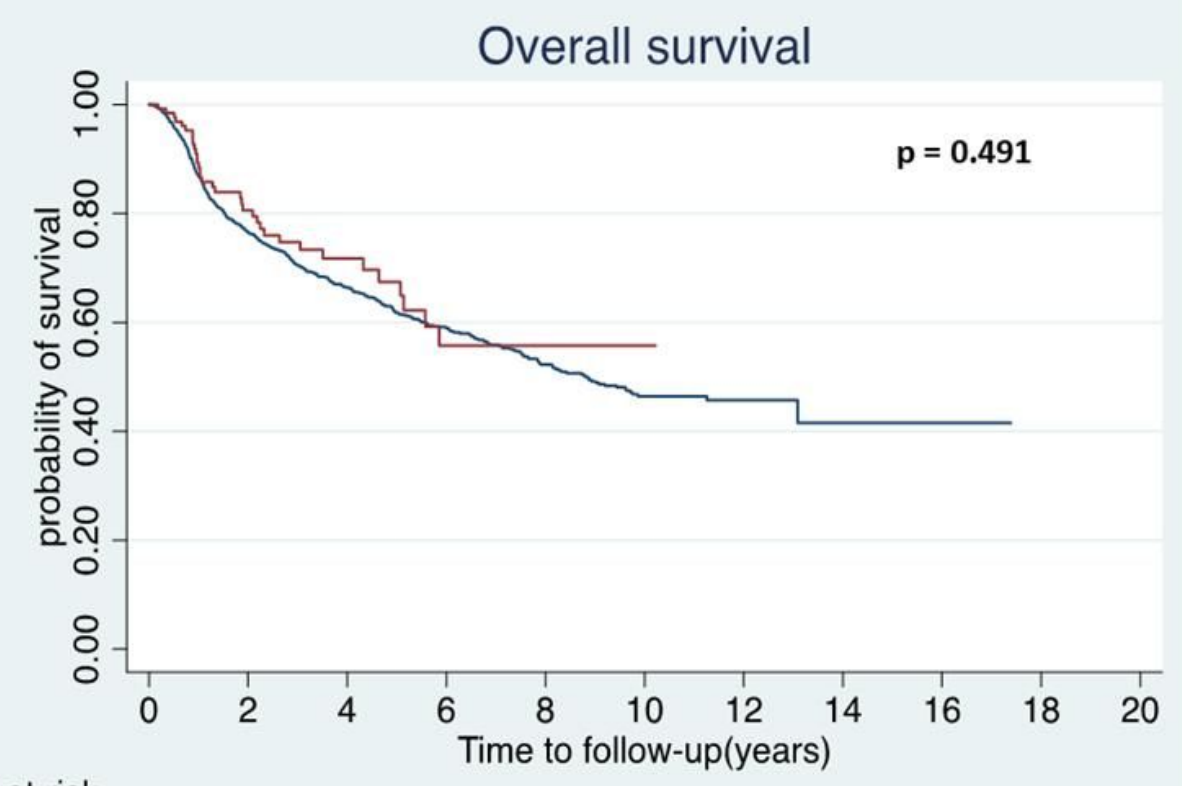

Number at risk

$\begin{array}{lllllllllll}\text { Male } 1846 & 899 & 556 & 366 & 246 & 128 & 27 & 5 & 1 & 0 & 0\end{array}$

Female 133

72

39

13

\begin{tabular}{|l|}
- Male \\
\end{tabular}

Figure 1 
Kaplan-Meier overall survival curve between genders (log rank test: $p=0.491$ )

Figure 2.

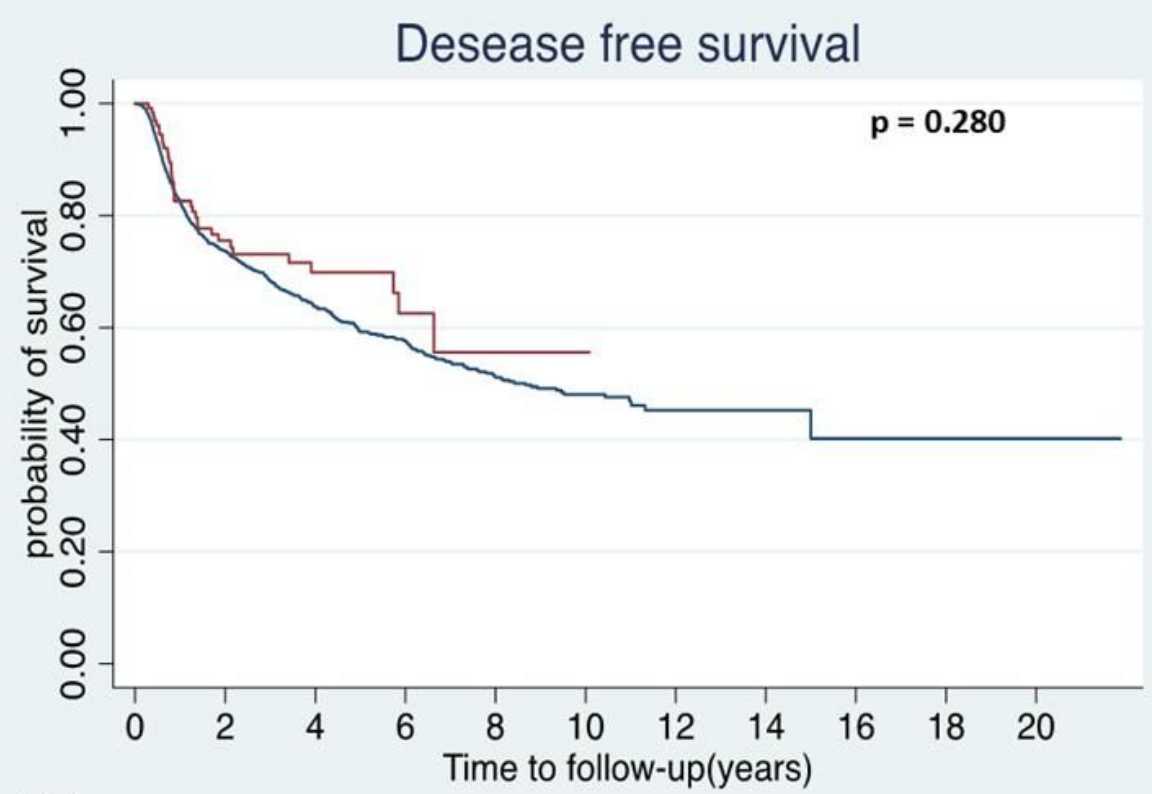

Number at risk

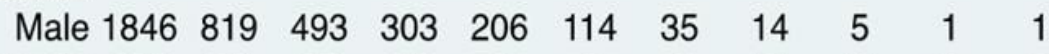

Female $\begin{array}{lllllllllll}133 & 66 & 37 & 14 & 6 & 2 & 0 & 0 & 0 & 0 & 0\end{array}$

\begin{tabular}{lll}
\hline Male & Female \\
\hline
\end{tabular}

\section{Figure 2}

Kaplan-Meier disease-free survival curve between genders (log rank test: $p=0.280$ ) 